\title{
Electrical Control of the Zeeman Spin Splitting in Two-Dimensional Hole Systems
}

\author{
E. Marcellina, ${ }^{1}$ A. Srinivasan, ${ }^{1}$ D. S. Miserev, ${ }^{1}$ A. F. Croxall, ${ }^{2}$ D. A. Ritchie, ${ }^{2}$ I. Farrer, ${ }^{2}$ O. P. Sushkov, ${ }^{1}$ \\ Dimitrie Culcer, ${ }^{1}$ and A. R. Hamilton ${ }^{1}$ \\ ${ }^{1}$ School of Physics, The University of New South Wales, Sydney 2052, Australia \\ ${ }^{2}$ Cavendish Laboratory, University of Cambridge, J. J. Thomson Avenue, Cambridge CB3 OHE, United Kingdom
}

(Received 28 March 2018; published 15 August 2018; corrected 17 August 2018)

\begin{abstract}
Semiconductor holes with strong spin-orbit coupling allow all-electrical spin control, with broad applications ranging from spintronics to quantum computation. Using a two-dimensional hole system in a gallium arsenide quantum well, we demonstrate a new mechanism of electrically controlling the Zeeman splitting, which is achieved through altering the hole wave vector $k$. We find a threefold enhancement of the in-plane $g$-factor $g_{\|}(k)$. We introduce a new method for quantifying the Zeeman splitting from magnetoresistance measurements, since the conventional tilted field approach fails for two-dimensional systems with strong spin-orbit coupling. Finally, we show that the Rashba spin-orbit interaction suppresses the in-plane Zeeman interaction at low magnetic fields. The ability to control the Zeeman splitting with electric fields opens up new possibilities for future quantum spin-based devices, manipulating non-Abelian geometric phases, and realizing Majorana systems in $p$-type superconductor systems.
\end{abstract}

DOI: 10.1103/PhysRevLett.121.077701

The spin-orbit interaction couples a particle's spin to its motion, as described by the Hamiltonian $H_{\mathrm{SO}}=$ $\frac{1}{2} \mu_{B} \boldsymbol{\sigma} \cdot \boldsymbol{B}_{\text {eff }}(\boldsymbol{k})$, where $\boldsymbol{\sigma}, \mu_{B}, \boldsymbol{B}_{\text {eff }}$, and $\boldsymbol{k}$ represent the Pauli matrices, the Bohr magneton, the effective spin-orbit magnetic field, and the motion wave vector, respectively [1]. The effective magnetic field $\boldsymbol{B}_{\text {eff }}$ emerges from a relativistic transformation that occurs when a particle with spin $\boldsymbol{\sigma}$ is moving with wave vector $\boldsymbol{k}$ with respect to an electric field $\boldsymbol{F}$. The spin-orbit interaction has applications in spintronic devices and spin-based quantum computing, which rely on controlling spin via external electric fields $\boldsymbol{F}$ [2-13].

Here we report a new mechanism for electrically manipulating spin through the Zeeman interaction in two-dimensional (2D) holes. Unlike in electrons, the Zeeman splitting in holes is highly anisotropic, with the out-of-plane $g$-factor $g_{z z}$ much larger than the in-plane one $\left(g_{z z} \gg g_{\|}\right)$, and $g_{\|}$strongly dependent on $k$. The ability to electrically control $g_{\|}$is not only valuable for applications in spintronics and quantum computing, but also for engineering non-Abelian geometric phases [14,15]. Moreover, in a hybrid semiconductor-superconductor system that can host Majorana fermions [16-22], a high tunability of the $g$ factor is desirable, as it is then possible to use a magnetic field to drive the system from the trivial to the topological regime without quenching the superconductivity needed to support the Majorana mode.

Previous methods of tuning the $g$ factor in 2D systems relied on shifting the wave function from one material to another, either by pushing it across a heterointerface or by using a graded composition quantum well [23]. Here we adopt a different approach, in which the $g$ factor is controlled not by shifting the wave function but by tuning the Fermi wave vector $k_{F}$ of a $2 \mathrm{D}$ hole system. Owing to the spin-3/2 nature of the 2D holes [24], increasing $k$ enhances the mixing between the occupied heavy hole and unoccupied light hole subbands, which then dramatically alters the in-plane $g$-factor $g_{\|}$. It is difficult to detect this variation of $g_{\|}$with optical methods, since these detect bound excitons with a small and fixed $k$ [25-27]. Instead we introduce a new approach based on magnetotransport in crossed magnetic fields, which shows that the spin splitting is linear in applied in-plane magnetic field and can be varied by $300 \%$ as $k_{F}$ is increased.

Our experiment was performed using an undoped $25 \mathrm{~nm}$ gallium arsenide (GaAs) quantum well sandwiched between $300 \mathrm{~nm} \mathrm{Al} \mathrm{Al}_{0.33} \mathrm{Ga}_{0.67}$ As layers and grown along the (001) direction. Metal top and back gates supplied the $2 \mathrm{D}$ carriers and allowed the quantum well symmetry to be tuned arbitrarily [28]. The magnetotransport measurements were performed in a dilution refrigerator at a base temperature of $\sim 30 \mathrm{mK}$. The sample was mounted on an in situ rotation system with an accuracy of $\pm 0.01^{\circ}$ [29]. Electrical measurements were carried out in the standard four-terminal configuration with a source-drain current of $5 \mathrm{nA}$ and a lockin frequency of $17 \mathrm{~Hz}$. The maximum 2D hole mobility was $1.5 \times 10^{6} \mathrm{~cm}^{2} \mathrm{~V}^{-1} \mathrm{~s}^{-1}$ at density $p=2.6 \times 10^{11} \mathrm{~cm}^{-2}$.

To demonstrate the $k$ dependence of the Zeeman spin splitting, we tune the front and back gate electric fields $\left(\vec{F}_{\text {front }}, \vec{F}_{\text {back }}\right)$ on the quantum well to alter $p$ (and hence $k_{F}$ ), while keeping the wave function in the quantum well center [Fig. 1(a)]. That is, we set the quantum well to 



FIG. 1. (a) Schematic of the GaAs hole quantum well used in this Letter, where $\psi_{H 1}$ is the heavy hole wave function and $V(z)$ is the confinement potential controlled via electric fields $\vec{F}_{\text {front }}$ and $\vec{F}_{\text {back }}$ applied using the front and back gates, respectively. Here, the total electric field $\vec{F}=F_{z} \hat{z} \equiv\left(\vec{F}_{\text {front }}+\vec{F}_{\text {back }}\right) / 2=0$. (b) The device is tilted at an angle $\theta$ with respect to the applied magnetic field $\mathbf{B}$. (c)-(e) Magnetoresistance oscillations $\rho_{x x}$ of the 2D holes in a symmetric quantum well at various tilt angles for three hole densities $p$. Because of the in-plane magnetic field, a resistance minimum gradually evolves into a maximum or vice versa, for example, at $p=1 \times 10^{11} \mathrm{~cm}^{-2}$ and $\nu=15$ for angles $\theta=0^{\circ}, 63.5^{\circ}, 78.9^{\circ}$ (see dashed box). (d)-(e) The effect of the in-plane magnetic field on the resistance becomes more pronounced as the $2 \mathrm{D}$ hole density increases: the transition between a resistance minimum and maximum occurs at smaller angles at higher densities (see dashed box). The traces have been offset for clarity.

be inversion symmetric, so that the net electric field $\vec{F} \equiv$ $F_{z} \hat{z} \equiv\left(\vec{F}_{\text {front }}+\vec{F}_{\text {back }}\right) / 2$ across the quantum well is zero, and there is no Rashba spin-orbit interaction $[1,30]$ (see Sec. S1 of the Supplemental Material [31]). We then introduce an in-plane magnetic field $B_{\|}$to cause an inplane Zeeman spin splitting. To measure the change in spin splitting as a function of $B_{\|}$, we apply a small $B_{z}$ to cause Shubnikov-de Haas oscillations, from which we measure the area of the Fermi surface. To this end, we tilt the sample at an angle $\theta$ with respect to the total magnetic field $\boldsymbol{B}$ [Fig. 1(b)]. The Shubnikov-de Haas oscillations at various $\theta$ for three different densities are shown in Figs. 1(c)-1(e). At $\theta=0^{\circ}$, for $0 \leq B_{z} \leq 0.25 \mathrm{~T}$, there is only one period of magnetoresistance oscillations $\rho_{x x}$, corresponding to a single spin-degenerate Fermi surface, with $\rho_{x x}$ minima occurring only at even filling factors $\nu_{\text {even }}$. At higher $B_{z}$, the out-of-plane Zeeman splitting $\propto g_{z z} B_{z}$ becomes visible, with $\rho_{x x}$ minima also developing at odd filling factors $\nu_{\text {odd }}$. Applying an in-plane magnetic field lifts the spin degeneracy even at low $B_{z}$, so that $\rho_{x x}$ varies as a function of $\theta$ at a fixed $B_{z}$ (and hence filling factor $\nu$ ). The dashed box in Fig. 1(c) shows that the resistance maximum for $p=$ $1 \times 10^{11} \mathrm{~cm}^{-2}$ at $\nu=15$ and $\theta=0^{\circ}$ evolves into a minimum as $\theta$ is gradually increased. Comparison between Figs. 1(d) and 1(e) shows that, as $p$ is increased, progressively smaller $\theta$ are required to cause this evolution. For $p=2 \times 10^{11} \mathrm{~cm}^{-2}$, the resistance minimum at $\nu=28$ and $0^{\circ}$ evolves into a weak minimum when $\theta$ is increased to $78.6^{\circ}$ [see the dashed box in Fig. 1(d)]. By contrast, for $p=3 \times 10^{11} \mathrm{~cm}^{-2}$ [see the dashed box in Fig. 1(e)], a weak minimum already develops at $\theta=63.5^{\circ}$ at $\nu=28$. This comparison provides evidence that the in-plane Zeeman splitting is density dependent. In fact, it was predicted that the in-plane Zeeman splitting in heavy holes, up to second order perturbation theory, is given by [1]

$$
\begin{aligned}
E\left(k_{F \pm}\right) & =\frac{\hbar^{2}}{2 m^{*}} k_{F \pm}^{2} \pm Z \mu_{B} k_{F \pm}^{2} B_{\|} \\
& \equiv \frac{\hbar^{2}}{2 m^{*}} k_{F \pm}^{2} \pm \frac{g_{\|}\left(k_{F \pm}\right)}{2} \mu_{B} B_{\|},
\end{aligned}
$$

where $\mu_{B}$ is the Bohr magneton and the Zeeman prefactor $Z$ is detailed in Sec. S4. B of the Supplemental Material [39]. Thus, the effect of $B_{\|}$is equivalent to changing the effective mass for the two spin subbands.

In 2D electrons, the $g$ factor can be extracted from tilted magnetic field measurements by finding the angle $\theta_{c}$ at which the Zeeman energy $g \mu_{B} B$ equals half the cyclotron energy $(1 / 2) \hbar e B \cos \theta_{c} / m^{*}$ [41]. Assuming the electron effective mass $m^{*}$ is known and $g$ is isotropic $\left(g_{z z}=g_{\|}\right)$, the $g$ factor is given by $g=\left(m_{0} \cos \theta_{c} / m^{*}\right)$, where $m_{0}$ is the bare electron mass. However, this approach is not applicable for holes, as the $g$ factor is highly anisotropic $\left(g_{z z} \gg g_{\|}\right)$, and $m^{*}$ depends on $B_{\|}$[Eq. (1); see also Sec. S2 of the Supplemental Material [42]). Instead, here we examine the dependence of the magnetoresistance on $B_{\|}$ at a fixed $B_{z}$, and hence a fixed $\nu$, to measure the area of the spin-split Fermi surfaces. To increase the visibility of the $B_{\|}$-induced magnetoresistance features against the smooth background, we define a dimensionless in-plane magnetoresistance $\Delta \bar{\rho}_{x x}$ at a given $\nu$

$$
\Delta \bar{\rho}_{x x}=\Delta \bar{\rho}_{x x}\left(B_{\|}\right) \equiv \frac{\rho_{x x}^{\nu}-\rho_{x x}^{\nu+1}}{\rho_{x x}^{\nu}+\rho_{x x}^{\nu+1}} .
$$



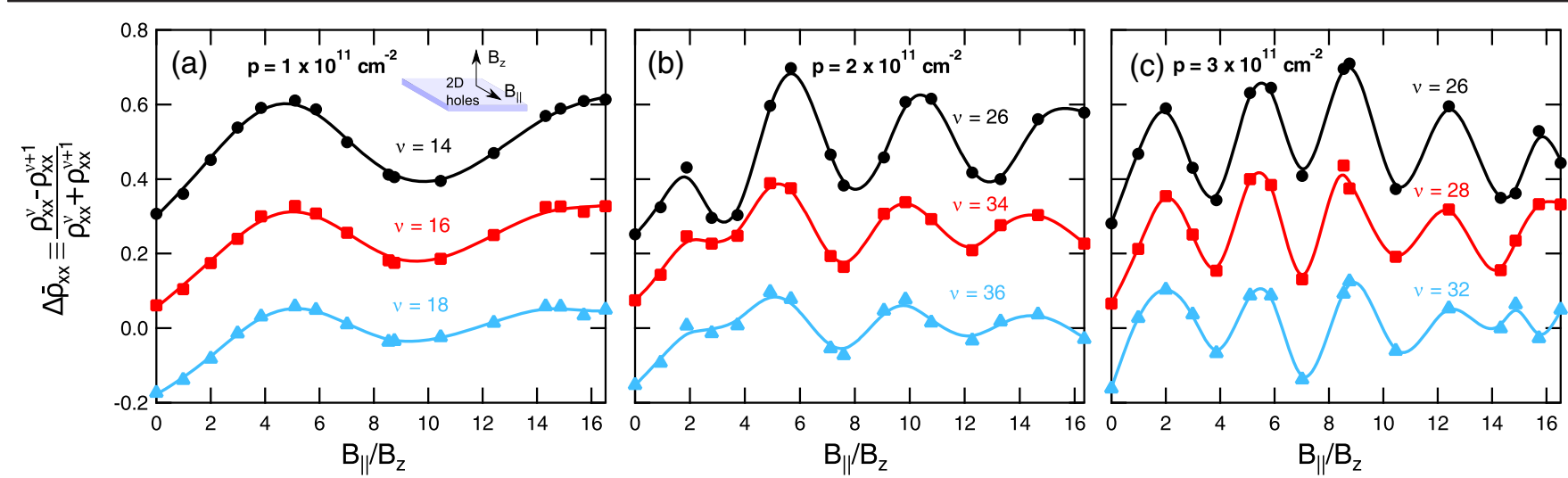

(d)

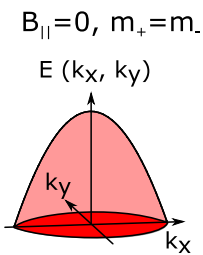

(g) $\mathrm{E}$

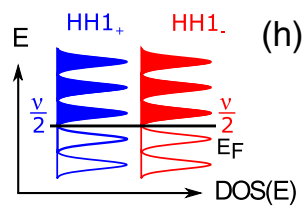

e) $B_{\| 1}=B_{1}, m_{+}>m$

$E\left(k_{x}, k_{y}\right)$

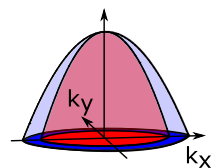

(h) $\mathrm{E}$

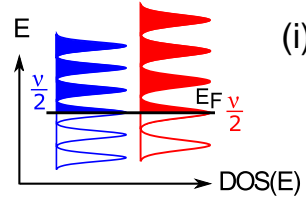

(i)

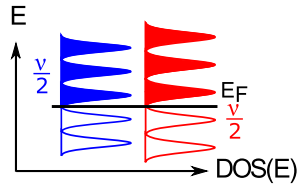

(f) $B_{\|}=2 B_{1}, m_{+}>m$.

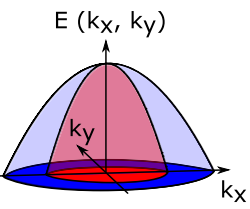

(j) $\Delta \bar{p}_{x x}$

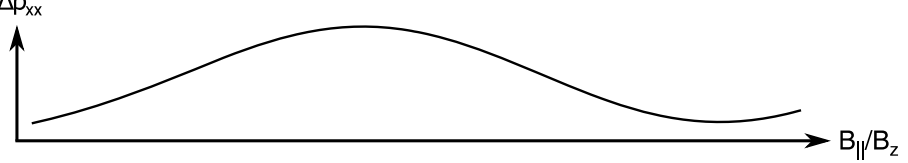

(k)

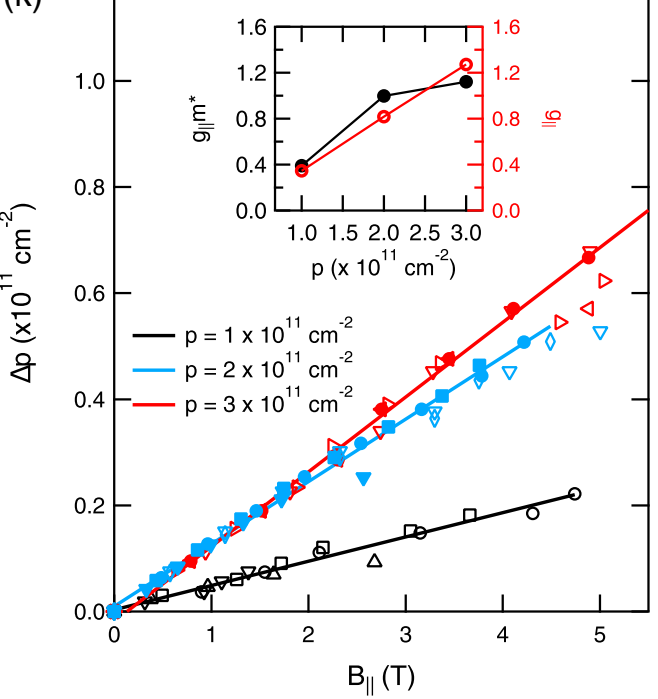

FIG. 2. (a)-(c) Resistance oscillations $\Delta \bar{\rho}_{x x}=\Delta \bar{\rho}_{x x}\left(B_{\|}\right) \equiv\left(\rho_{x x}^{\nu}-\rho_{x x}^{\nu+1}\right) /\left(\rho_{x x}^{\nu}+\rho_{x x}^{\nu+1}\right)$ at various filling factors $\nu$ for different $p$. (d)-(f) Schematic of the energy band dispersion $E\left(k_{x}, k_{y}\right)$, showing how the $k$-dependent Zeeman splitting, where $k \equiv \sqrt{k_{x}^{2}+k_{y}^{2}}$, changes the effective masses $m_{+}$and $m_{-}$in the two bands. (g)-(i) Schematic of the Landau levels corresponding to the band structure in (d)-(f) for an even $\nu$. The shaded regions refer to the filled Landau levels. As $B_{\|}$is increased from $0 \mathrm{~T}$ to $B_{\|}=B_{1}>0 \mathrm{~T}$, the Landau level spacing in the two spin-split bands changes due to the different masses. (j) The in-plane magnetoresistance $\Delta \bar{\rho}_{x x}$ arises from the change in the density of states at the Fermi energy as illustrated in (d)-(f). (k) The difference in the hole density of the two spin-split bands $\Delta p$ as a function of $B_{\|}$for different $p$, as indicated by the three different colors. (Inset) Shows $g_{\|} m^{*}$ (solid black line with solid circles) extracted using Eq. (1). Using the effective masses obtained from $6 \times 6 \boldsymbol{k} \cdot \boldsymbol{p}$ calculations [43], we find $g_{\|}$(solid red line with open circles), which increases linearly with density.

The magnetoresistance $\bar{\rho}_{x x}$ [Figs. 2(a)-2(c)] oscillates as a function of $B_{\|}$, with the frequency of the oscillations being independent of $\nu$. Furthermore, the frequency increases with $p$.

The physical mechanism underlying the density dependence of $\bar{\rho}_{x x}$ is illustrated in Figs. 2(d)-2(f). We first consider the energy dispersion $E\left(k_{x}, k_{y}\right)$ in the absence of $B_{z}$. When $B_{\|}=0$, the lowest heavy hole (HH) subbands $\left(\mathrm{HH} 1_{ \pm}\right)$are degenerate [Fig. 2(d)]. The effect of $B_{\|}$on the energy dispersion is remarkably different for $2 \mathrm{D}$ holes than electrons. For 2D electrons, $B_{\|}$splits all $k$ states by $\Delta E_{Z}=g \mu_{B} B_{\|}$, whereas for $2 \mathrm{D}$ holes, $B_{\|}$causes a $k$ dependent splitting of the $\mathrm{HH} 1_{+}$and $\mathrm{HH} 1_{-}$bands, so that $m_{+} \neq m_{-}$[Fig. 2(e)], where $m_{ \pm}$is the effective mass of the $\mathrm{HH} 1_{ \pm}$bands. The $k$-dependent spin splitting increases with $B_{\|}$, and the corresponding effective masses $m_{+}$and $m_{-}$ diverge further [Fig. 2(f)] with $B_{\|}$. When $B_{z}$ is finite, Landau levels form, where the energy separation between the Landau levels depends on the effective mass and hence on $B_{\|}$. When $B_{\|}=0$, the $\mathrm{HH}_{ \pm}$Landau levels are spin degenerate, $m_{+}=m_{-}$, and the number of occupied Landau levels is identical in the $\mathrm{HH}_{+}$and $\mathrm{HH} 1_{-}$subbands $\left(\nu_{+}=\nu_{-}\right)$[Fig. 2(g)]. When $B_{\|} \neq 0, m_{+}$diverges from $m_{-}$, changing the spacing of Landau levels in the $\mathrm{HH} 1_{+}$and HH1_ bands. Thus, the density of states at the Fermi energy $E_{F}$ as $\nu_{+}$and $\nu_{-}$changes with $B_{\|}$[Figs. 2(h) and 2(i)]. 
Assuming that the change $\Delta \nu_{ \pm}$in the occupancy of the $\mathrm{HH} 1_{ \pm}$Landau levels is much smaller than $\nu\left(\Delta \nu_{ \pm} / \nu \ll 1\right)$, the Landau level occupation changes in pairs, i.e., $\Delta \nu_{+}=$ $-\Delta \nu_{-}$[Fig. 2(g)].

The change in the density of states at $E_{F}$ causes $\Delta \bar{\rho}_{x x}$ to oscillate as a function of $B_{\|}$at a fixed $B_{z}$. When $E_{F}$ coincides (does not coincide) with a Landau level, a maximum (minimum) in $\Delta \bar{\rho}_{x x}$ develops [Fig. 2(j)]. Since one oscillation period of $\Delta \bar{\rho}_{x x}$ corresponds to $\left|\Delta \nu_{ \pm}\right|=1$, the spin splitting after moving $n \bar{\rho}_{x x}\left(B_{\|}\right)$resistance peaks away from the symmetry point is given by

$$
\frac{\nu / 2+n \Delta \nu_{ \pm}}{\nu}=\frac{p_{ \pm}}{p}
$$

where $p_{ \pm}$is the spin-split densities. Note that although Eq. (3) is exact for parabolic and isotropic bands (see Sec. S2 of the Supplemental Material [42]), it also holds for nonparabolic and/or anisotropic bands as long as $\Delta \nu_{ \pm} / \nu \ll 1$ (see Secs. S3 and S4 of the Supplemental Material [44]). We remark that the resistance oscillations $\Delta \bar{\rho}_{x x}\left(B_{\|}\right)$are analogous to Shubnikov-de Haas oscillations: in conventional Shubnikov-de Haas oscillations, the Landau level spacing $e B_{z} / m^{*}$ is controlled by varying $B_{z}$, whereas the oscillations $\Delta \bar{\rho}_{x x}\left(B_{\|}\right)$are caused by varying $m_{ \pm}$.

We use Eq. (3) to extract from $\Delta \bar{\rho}_{x x}\left(B_{\|}\right)$the change in the area of the spin-split Fermi surfaces, and hence the spin splitting $\Delta p$, as a function of $B_{\|}$. We obtain $\Delta p$ for multiple values of $\nu$, as indicated by the symbols in Fig. 2(k). The spin splitting is independent of $\nu$ and increases linearly with $B_{\|}$. To obtain $g_{\|}=g_{\|}\left(k_{F}\right)$ from $\Delta p$, we use the dispersion relation in Eq. (1) and obtain $Z \mu_{B}=1.37,1.88$, $1.95 \times 10^{-18} \mathrm{meV} \mathrm{m}^{2} \mathrm{~T}^{-1}$ and $g_{\|}\left(k_{F}\right) m^{*} / m_{0}=0.39,0.99$, and 1.18 for $p=1,2$, and $3 \times 10^{11} \mathrm{~cm}^{-2}$, respectively [shown by the solid black line and solid circles in the inset to Fig. 2(k)]. Using the effective masses obtained from $6 \times 6 \boldsymbol{k} \cdot \boldsymbol{p}$ calculations [43], i.e., $m^{*}=1.13,1.22$, and 0.88 $m_{0}$ for $p=1,2$, and $3 \times 10^{11} \mathrm{~cm}^{-2}$, respectively, we find $g_{\|}=0.34,0.82$, and 1.26 [shown by the solid red line and open circles in the inset to Fig. 2(k)]. These values of $g_{\|}$are of the same order of magnitude as the predicted values for 2D hole systems [46,47]. It is also interesting to note that the values of $g_{\|}$we measure here are consistent with the experimental results for quasi-one-dimensional hole systems [48-50].

Finally, we investigate the interplay of Rashba and Zeeman interactions by repeating the tilted magnetic field measurements with a finite electric field $F_{z}$ applied across the quantum well and a fixed density of $p=$ $2 \times 10^{11} \mathrm{~cm}^{-2}$. Figures 3(a) and 3(b) show Shubnikovde Haas oscillations for $F_{z}=0$ and $0.43 \mathrm{MV} / \mathrm{m}$. When the quantum well is symmetric $\left(F_{z}=0 \mathrm{MV} / \mathrm{m}\right)$, the Rashba

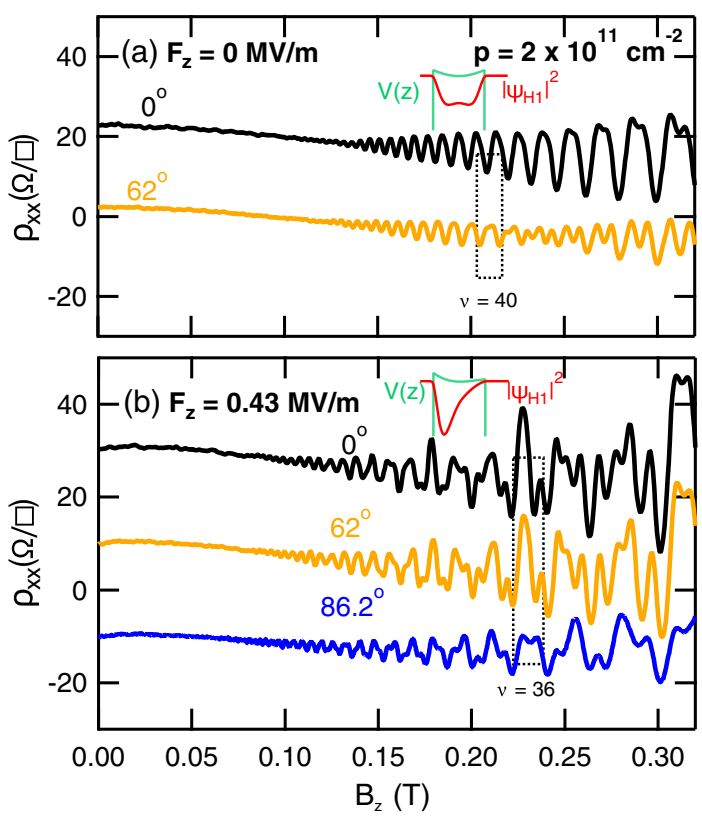

FIG. 3. (a) In the absence of Rashba splitting $\left(F_{z}=0 \mathrm{MV} / \mathrm{m}\right)$, the magnetoresistance minima change into maxima (or vice versa) when $\theta$ is increased from $0^{\circ}$ to $62^{\circ}$, as highlighted in the dashed box for $\nu=40$. (b) At $F_{z}=0.43 \mathrm{MV} / \mathrm{m}$, the Shubnikov-de Haas oscillations are far less affected by $\theta$ : when $\theta$ is increased from $0^{\circ}$ to $62^{\circ}$ the positions of the minima and maxima remain almost unchanged, and only when $\theta$ is large $\left(\theta>80^{\circ}\right)$ do the resistance minima evolve into maxima (the dashed box highlights $\nu=36$ ). Here, $p=2 \times 10^{11} \mathrm{~cm}^{-2}$. The shape of the potential profile and ground state envelope wave function are shown in green and red. The traces are offset for clarity.

splitting is zero, and the magnetoresistance at $\theta=0^{\circ}$ shows a single oscillation period at low $B_{z}$, with each Landau level being doubly degenerate. In this case, $B_{\|}$has a strong influence on the Shubnikov-de Haas oscillations, as shown by the dashed box. By contrast, when $F_{z}$ is large $\left(F_{z}=0.43 \mathrm{MV} / \mathrm{m}\right)$, the magnetoresistance at $\theta=0$ shows a beating due to the Rashba splitting even at $B_{\|}=0$. A Fourier transform of the $\theta=0^{\circ}$ oscillations shows a large Rashba splitting of $\Delta p / p=40 \%$ (see Sec. S1 of the Supplemental Material [31]). This Rashba interaction dramatically suppresses the effects of $B_{\|}$on the Shubnikov-de Haas oscillations: the positions of maxima and minima for $\theta=0^{\circ}$ and $\theta=62^{\circ}$ are practically identical and only at high tilt angles, $\theta>80^{\circ}$, do the oscillation minima evolve into maxima or vice versa.

It is challenging to perform a complete quantitative analysis to extract $\Delta p$ and $g_{\|}$when both Rashba and Zeeman interactions are finite. The method of counting the periodic Landau level population or depopulation becomes invalid when $\Delta \nu / \nu \ll 1$ is violated, which is the case with $F_{z}=0.43 \mathrm{MV} / \mathrm{m}$. There is currently no analytical or numerical modeling of 2D holes under the Rashba spinorbit interaction in tilted magnetic field configuration 
against which we can compare our data. Such calculations are extremely challenging due to the combination of quantum confinement, the four-component hole spinor [51], heavy hole and light hole coupling, as well as the simultaneous inclusion of $B_{z}$ and $B_{\|}$[52]. To get a qualitative understanding of Fig. 3, we present a minimal model for the spin splitting when both Rashba and in-plane Zeeman interactions are present at $B_{z}=0 \mathrm{~T}$ (see Sec. S5 of the Supplemental Material [42]). The suppression of $g_{\|}$in Fig. 3 is due to the Rashba interaction dominating over the in-plane Zeeman interaction. In this limit, the effect of $B_{\|}$is to offset the center of the Fermi surfaces from $k=0$, without changing the areas appreciably. This is because the Rashba and in-plane Zeeman interactions have similar functional forms, i.e., $\left(k_{-}^{3} \sigma_{+}+k_{+}^{3} \sigma_{-}\right)$and $\left(k_{-}^{2} B_{-} \sigma_{+}+k_{+}^{2} B_{+} \sigma_{-}\right)$, respectively. We note that, in the opposite limit, where the in-plane Zeeman interaction is much larger than the Rashba interaction, $g_{\|}$reverts to its value at zero Rashba interaction [Fig. S3(a) of the Supplemental Material [42] ].

In summary, we have demonstrated all-electrical control of the in-plane Zeeman splitting of 2D holes by separately varying the $2 \mathrm{D}$ hole density $p$ and the electric field $F_{z}$ across the quantum well. We have developed a novel method to quantify the Zeeman splitting from the tilted magnetic field measurements. This new method can in principle be generalized to other $2 \mathrm{D}$ materials with an anisotropic $g$ factor $\left(g_{\|} \neq g_{z z}\right)$ as long as the confinement potential is inversion symmetric. By tracking the evolution of the Landau levels as a function of $B_{\|}$, we show that the in-plane Zeeman splitting of 2D holes is proportional to $p$ and that $g_{\|}$can be tripled. We have also shown that a strong $F_{z}$ can suppress $g_{\|}$, although a complete quantitative analysis for extracting $g_{\|}$in the case of finite $F_{z}$ is beyond the scope of the present Letter. The ability to electrically tune $g_{\|}$will be useful for designing spin-based devices, such as spin transistors, spin-orbit qubits, quantum logic gates, and hybrid superconductor-semiconductor systems hosting Majorana modes.

The authors thank Jo-Tzu Hung and Weizhe Liu for enlightening discussions. This work was supported by the Australian Research Council under the Discovery Projects scheme. We also acknowledge financial support from the EPSRC, UK, author I. F. from Toshiba Research Europe, and author A. F. C. from Trinity College, Cambridge.

[1] R. Winkler, Spin Orbit Coupling Effects in TwoDimensional Electron and Hole Systems (Springer-Verlag, Berlin, 2003).

[2] S. Datta and B. Das, Appl. Phys. Lett. 56, 665 (1990).

[3] I. Žutić, J. Fabian, and S. D. Sarma, Rev. Mod. Phys. 76, 323 (2004).

[4] D. Loss and D. P. DiVincenzo, Phys. Rev. A 57, 120 (1998).
[5] S. A. Wolf, D. D. Awschalom, R. A. Buhrman, J. M. Daughton, S. von Molnár, M. L. Roukes, A. Y. Chtchelkanova, and D. M. Treger, Science 294, 1488 (2001).

[6] D. D. Awschalom, L. C. Bassett, A. S. Dzurak, E. L. Hu, and J. R. Petta, Science 339, 1174 (2013).

[7] K. C. Nowack, F. H. L. Koppens, Y. V. Nazarov, and L. M. K. Vandersypen, Science 318, 1430 (2007).

[8] D. V. Bulaev and D. Loss, Phys. Rev. Lett. 98, 097202 (2007).

[9] S. Nadj-Perge, S. M. Frolov, E. P. A. M. Bakkers, and L. P. Kouwenhoven, Nature (London) 468, 1084 (2010).

[10] V. S. Pribiag, S. Nadj-Perge, S. M. Frolov, J. W. G. van den Berg, I. van Weperen, S. R. Plissard, E. P. A. M. Bakkers, and L. P. Kouwenhoven, Nat. Nanotechnol. 8, 170 (2013).

[11] R. Maurand, X. Jehl, D. K. Patil, A. Corna, H. Bohuslavskyi, R. Laviéville, L. Hutin, S. Barraud, M. Vinet, M. Sanquer, and S. De Franceschi, Nat. Commun. 7, 13575 (2016).

[12] J. Salfi, J. A. Mol, D. Culcer, and S. Rogge, Phys. Rev. Lett. 116, 246801 (2016).

[13] J.-T. Hung, E. Marcellina, B. Wang, A. R. Hamilton, and D. Culcer, Phys. Rev. B 95, 195316 (2017).

[14] J. C. Budich, D. G. Rothe, E. M. Hankiewicz, and B. Trauzettel, Phys. Rev. B 85, 205425 (2012).

[15] T. Li, L. A. Yeoh, A. Srinivasan, O. Klochan, D. A. Ritchie, M. Y. Simmons, O. P. Sushkov, and A. R. Hamilton, Phys. Rev. B 93, 205424 (2016).

[16] J. Alicea, Y. Oreg, G. Refael, F. von Oppen, and M. P. A. Fisher, Nat. Phys. 7, 412 (2011).

[17] R. M. Lutchyn, T. D. Stanescu, and S. Das Sarma, Phys. Rev. Lett. 106, 127001 (2011).

[18] L. Mao, M. Gong, E. Dumitrescu, S. Tewari, and C. Zhang, Phys. Rev. Lett. 108, 177001 (2012).

[19] V. Mourik, K. Zuo, S. M. Frolov, S. R. Plissard, E. P. A. M. Bakkers, and L.P. Kouwenhoven, Science 336, 1003 (2012).

[20] L. P. Rokhinson, X. Liu, and J. K. Furdyna, Nat. Phys. 8, 795 (2012).

[21] S. Vaitieknas, M. T. Deng, J. Nygård, P. Krogstrup, and C. M. Marcus, arXiv:1710.04300 [Phys. Rev. Lett (to be published)].

[22] J. Liang and Y. Lyanda-Geller, Phys. Rev. B 95, 201404 (2017).

[23] G. Salis, Y. Kato, K. Ensslin, D. C. Driscoll, A. C. Gossard, and D. D. Awschalom, Nature (London) 414, 619 (2001).

[24] J. M. Luttinger, Phys. Rev. 102, 1030 (1956).

[25] M. Syperek, D. R. Yakovlev, A. Greilich, J. Misiewicz, M. Bayer, D. Reuter, and A. D. Wieck, Phys. Rev. Lett. 99, 187401 (2007).

[26] M. Kugler, T. Andlauer, T. Korn, A. Wagner, S. Fehringer, R. Schulz, M. Kubová, C. Gerl, D. Schuh, W. Wegscheider, P. Vogl, and C. Schüller, Phys. Rev. B 80, 035325 (2009).

[27] C. Gradl, M. Kempf, D. Schuh, D. Bougeard, R. Winkler, C. Schüller, and T. Korn, Phys. Rev. B 90, 165439 (2014).

[28] A. F. Croxall, B. Zheng, F. Sfigakis, K. Das Gupta, I. Farrer, C. A. Nicoll, H. E. Beere, and D. A. Ritchie, Appl. Phys. Lett. 102, 082105 (2013).

[29] L. A. Yeoh, A. Srinivasan, T. P. Martin, O. Klochan, A. P. Micolich, and A. R. Hamilton, Rev. Sci. Instrum. 81, 113905 (2010).

[30] Y. A. Bychkov and E. I. Rashba, J. Phys. C 17, 6039 (1984). 
[31] See Supplemental Material at http://link.aps.org/ supplemental/10.1103/PhysRevLett.121.077701 for details on tuning the quantum well asymmetry, which includes Refs. [32-38].

[32] G. Dresselhaus, Phys. Rev. 100, 580 (1955).

[33] P. T. Coleridge, R. Stoner, and R. Fletcher, Phys. Rev. B 39, 1120 (1989).

[34] R. Winkler, S. J. Papadakis, E. P. De Poortere, and M. Shayegan, Phys. Rev. Lett. 84, 713 (2000).

[35] S. Keppeler and R. Winkler, Phys. Rev. Lett. 88, 046401 (2002).

[36] B. Habib, M. Shayegan, and R. Winkler, Semicond. Sci. Technol. 24, 064002 (2009).

[37] T. Li, B. Horovitz, and O. P. Sushkov, Phys. Rev. B 93, 235316 (2016).

[38] E. Marcellina, A. R. Hamilton, R. Winkler, and D. Culcer, Phys. Rev. B 95, 075305 (2017).

[39] See Supplemental Material at http://link.aps.org/ supplemental/10.1103/PhysRevLett.121.077701 for additional details and calculations, which includes Ref. [40].

[40] A. Baldereschi and N. Lipari, Phys. Rev. B 8, 2697 (1973).

[41] F. F. Fang and P. J. Stiles, Phys. Rev. 174, 823 (1968).

[42] See Supplemental Material at http://link.aps.org/ supplemental/10.1103/PhysRevLett.121.077701 for detailed calculations.

[43] S. Birner, T. Zibold, T. Andlauer, T. Kubis, M. Sabathil, A. Trellakis, and P. Vogl, IEEE Trans. Electron Devices 54, 2137 (2007).
[44] See Supplemental Material at http://link.aps.org/ supplemental/10.1103/PhysRevLett.121.077701 for a detailed derivation of Eq. (3), which includes Ref. [45].

[45] O. Gunawan, Y. P. Shkolnikov, E. P. De Poortere, E. Tutuc, and M. Shayegan, Phys. Rev. Lett. 93, 246603 (2004).

[46] T. Kernreiter, M. Governale, and U. Zülicke, Phys. Rev. Lett. 110, 026803 (2013).

[47] D. S. Miserev and O. P. Sushkov, Phys. Rev. B 95, 085431 (2017).

[48] D. S. Miserev, A. Srinivasan, O. A. Tkachenko, V. A. Tkachenko, I. Farrer, D. A. Ritchie, A. R. Hamilton, and O. P. Sushkov, Phys. Rev. Lett. 119, 116803 (2017).

[49] F. Nichele, A. N. Pal, R. Winkler, C. Gerl, W. Wegscheider, T. Ihn, and K. Ensslin, Phys. Rev. B 89, 081306(R) (2014).

[50] A. Srinivasan, D. S. Miserev, K. L. Hudson, O. Klochan, K. Muraki, Y. Hirayama, D. Reuter, A. D. Wieck, O.P. Sushkov, and A. R. Hamilton, Phys. Rev. Lett. 118, 146801 (2017).

[51] R. Winkler, Phys. Rev. B 71, 113307 (2005).

[52] L. A. Yeoh, A. Srinivasan, O. Klochan, R. Winkler, U. Zülicke, M. Y. Simmons, D. A. Ritchie, M. Pepper, and A. R. Hamilton, Phys. Rev. Lett. 113, 236401 (2014).

Correction: The list of author names has been reordered. 